\title{
Corrigendum
}

\section{Corrigendum to "Evaluation of the electric force in electrophoresis" [J. Colloid Interface Sci. 305 (2007) 324-329]}

\author{
Jyh-Ping Hsu *, Li-Hsien Yeh, Ming-Hong Ku
}

Department of Chemical Engineering, National Taiwan University, Taipei, Taiwan 10617

Available online 23 January 2007

On page 326, Eq. (31) should be corrected as

$0=\eta \nabla^{2} \mathbf{u}-\nabla p+\varepsilon \nabla^{2} \Psi \nabla \Psi$.

On page 327, Eqs. (52), (53), (55), and (56) should be respectively corrected as

$\mathbf{n} \cdot \nabla \Psi_{1}=\frac{e N_{\mathrm{S}} / \varepsilon}{1+\frac{\left[\mathrm{H}^{+}\right]_{\mathrm{b}}}{K_{\mathrm{a}}} \exp \left(-\frac{e \Psi_{1}}{k_{\mathrm{B}} T}\right)}$,

$$
\mathbf{n} \cdot \nabla^{*} \Psi_{1}^{*}=\frac{A}{1+B \exp \left(-\Psi_{1}^{*}\right)},
$$

$$
\mathbf{n} \cdot \nabla \Psi_{1}=\frac{e N_{\mathrm{S}} / \varepsilon}{\left\{1+\left[\mathrm{H}^{+}\right]_{\mathrm{b}} / K_{\mathrm{a}}\right\}}+\frac{\left(e^{2} N_{\mathrm{S}} / \varepsilon k_{\mathrm{B}} T\right)\left\{\left[\mathrm{H}^{+}\right]_{\mathrm{b}} / K_{\mathrm{a}}\right\}}{\left\{1+\left[\mathrm{H}^{+}\right]_{\mathrm{b}} / K_{\mathrm{a}}\right\}^{2}} \Psi_{1},
$$

$\mathbf{n} \cdot \nabla^{*} \Psi_{1}^{*}=\frac{A}{1+B}+\frac{A B}{[1+B]^{2}} \Psi_{1}^{*}$. 